Les Cahiers du Gres

\section{Ethnicité, nation et sexe-genre}

\section{Danielle Juteau}

Volume 1, numéro 1, automne 2000

URI : https://id.erudit.org/iderudit/009418ar

DOI : https://doi.org/10.7202/009418ar

Aller au sommaire du numéro

Éditeur(s)

Groupe de recherche ethnicité et société

CEETUM

ISSN

1499-0431 (imprimé)

1499-044X (numérique)

Découvrir la revue

Citer cet article

Juteau, D. (2000). Ethnicité, nation et sexe-genre. Les Cahiers du Gres, 1(1),

53-57. https://doi.org/10.7202/009418ar
Les Cahiers du Gres Série Diversité urbaine Groupe de Recherche Ethnicte et Societs 


\section{Danielle JUTEAU}

Professeure titulaire

Département de sociologie

Titulaire de la Chaire en relations ethniques

Membre

Groupe de recherche ethnicité et société

Université de Montréal

\section{ETHNICITÉ, NATION ET SEXE- GENRE*}

Depuis une vingtaine d'années, des chercheurs, féministes pour la plupart, ont infléchi les déb ats sur l'ethnicité, la nation et le sexe-genre. Un double objectif se dégage de leurs travaux: 1) examiner la manière dont s'entrecroisent et s'articulent le genre, l'ethnicité, la nation ainsi que la " race ";2) appréhender le rapport spécifique des femmes au groupe ethnique et à la nation. Or ces débats sont indissociables de la définition même de ces concepts.

\section{Ethnicité}

Le concept d'ethnicité est récent. II remonte à 1942 lorsque les Américains Lloyd Warner et Paul Lunt I'utilisent dans leurs célèbres travaux sur Yankee City (The Social life of a Modern Community, Yankee City Series, vol.1, 1941, New Haven, Yale University Press, 460p. ; The Status System of a Modern Community, Yankee City Series, vol.2, 1973 [1942], Wesport, Conn., Greenwood Press, 246p.). L'ethnicité est ici une caractéristique qui à l'instar de l'âge, du sexe et de la religion, modifie le système social tout en étant en retour modifiée par ce dernier. À côté de cette définition, qui est inclusive, on retrouve une conception plus restreinte du terme car pour plusieurs auteurs, le groupe dominant ne constitue pas à proprement parler un groupe ethnique. En effet, le nom grec ethnikos renvoie étymologiquement aux gentils, aux infidèles, donc aux autres. Ce n'est qu'aux 18e et 19 e siècles que réapparaît le terme ethnique pour désigner une spécificité liée à la " race " ou à la nation (Werner Sollors, Beyond Ethnicity. Consent and Descent in American Culture, New York et Oxford, Oxford University Press, 1986, 294p.).

Si dans les pays colonisateurs tels la France et l'Angleterre les scientifiques se sont gardés d'aborder un objet qui fut trop longtemps racialisé, - on se souviendra que le terme ethnie dans le discours colonial renvoie à l'idée de race-, l'étude des relations ethniques connaît en Amérique du Nord un essor considérable dès le début du ving tième siècle. Aux approches primordialistes et souvent essentialistes axées sur la description de traits culturels statiques et figés ont succédé depuis la fin des années soixante des analyses davantage relationnelles. Ces dernières envisagent l'ethnicité comme l'expression d'intérêts communs ou le reflet d'antagonismes économiques, ou encore comme système culturel ou forme d'interaction sociale (Philippe Poutignat et Jocelyne Streiff-Fenart, Théories de l'ethnicité, Paris, PUF, 1995, 270p).

Dorénavant, l'ethnicité est conçue comme fluide et construite à l'intérieur de relations inégalitaires, comme un rapport social possédant une face externe, rapport à autrui et une face interne, rapport à une histoire et à une origine communes (Danielle Juteau, L'ethnicité et ses frontières, Montréal, Presses de l'Université de Montréal, 1999, 226p.). Les liens qui rassemblent les membres d'un groupe ethnique servent à fonder la communauté nationale, laquelle se caractérise par la présence d'un État ou d'un projet visant à son établissement.

\section{Nation}

Le concept de nation fait lui aussi et depuis fort longtemps, l'objet de multi- 
ples controverses théoriques et politiques. Si les querelles opposant divers penseurs d'orientation marxiste tels Otto Bauer et Karl Kautsky appartiennent à un autre siècle, la définition de la nation demeure contestée. Tandis que perdurent les débats entre marxistes et non marxistes, la nation est dorénavant pensée selon tel ou tel auteur, soit comme réalité subjective ou objective, soit comme forme idéologique ou comme forme politique moderne, soit comme communauté de destin, communauté imaginaire ou communauté imaginée (Benedict Anderson, Imagined Communities: Reflections on the Origin and Spread of Nationalism, Londres, Verso, 1991, 224p.).

Les travaux les plus récents récusent également toute définition substantialiste de la nation, selon laquelle les nations seraient des entités réelles. On y rejette tant l'approche essentialiste fondée sur la croyance en l'existence déterminante des racines et des origines que les nouvelles conceptions modernistes et constructivistes axées sur l'industrialisation, la modernisation, le développement inégal, la croissance des réseaux de communication et de transport ou tout autre autre facteur intégratif de l'État moderne, engendrant la nation. II faudrait plutôt, selon Rogers Brubaker (Nationalism reframed. Nationhood and the national question in the new Europe, Cambridge, Cambridge University Press, 1996, 202p.) se pencher sur la nation comme catégorie cognitive et socio-politique (nationhood) et comme événement contingent (nationness), mettant ainsi l'accent sur le contexte relationnel lui-même.

Enfin, on s'interroge aussi sur la valeur des divers modèles nationaux, naturaliste et organiciste, volontariste et politique (Dominique Schnapper, La France de I'Intégration. Sociologie de la Nation en 1990, Paris, PUF, 1991, 374p.) auxquels s'est ajoutée depuis une trentaine d'années une conception pluraliste et multiculturelle de l'État-nation.

\section{Genre et sexe}

Les recherches sur les rapports hommes-femmes ont connu une évolution semblable, en ce sens qu'on a délaissé l'examen de leurs attributs distinctifs pour se pencher sur le rapport social. L'analyse s'est déplacée de l'étude du sexe comme variable indépendante (le sexe biologique étant la source des différences observées entre les hommes et les femmes) et de la comparaison des rôles masculins et des rôles féminins, pour porter sur le genre comme construit social et sur l'acquisition de la masculinité et de la féminité. Très utilisé dans les milieux anglo-saxons, ce concept se voit remis en question par celles (Judith Butler, Gender Trouble : Feminism and the Subversion of Identity, Londres, Routledge, 1990, 172p.) qui rejettent aujourd'hui l'idée d'un lien obligatoire entre le sexe et le genre, chaque sexe pouvant choisir le genre qui lui convient.

D'autres approches, matérialistes, examinent l'appropriation du travail des femmes, de leur corps et de leur individualité. La mise à nu du sexage (Colette Guillaumin, "Pratique du pouvoir et idée de Nature (1) :L'appropriation des femmes ", Questions féministes, no.2 : 5-30, 1978), ce rapport constitutif de la classe des hommes et de la classe des femmes, dévoile que les catégories de sexe ne sont pas données mais construites. C'est le genre qui crée le sexe et non l'inverse.

\section{L'hétérogénéité de la catégorie " femmes"}

Cherchant à déconstruire I'homogénéité de la catégorie " femmes " et à tenir compte de la diversité de leurs situations, de leurs intérêts et de leurs identités, des penseures féministes introduisent, au sein de leurs analyses, d'autres dimensions sociales dont la classe et la " race ", à laquelle furent ajoutées l'ethnicité et la nation. 
Si les débats des années soixante-dix furent centrés, en France et en GrandeBretagne notamment, sur les intérêts divergents des femmes de la bourgeoisie et du prolétariat, ils ont ensuite porté, principalement dans les milieux anglophones, sur l'opposition entre femmes blanches et femmes de couleur. À partir des années quatre-vingt, des féministes noires et des pays colonisés taxent d'essentialisme le féminisme dominant, en l'occurrence celui des femmes blanches. Ce dernier postulerait une féminitude partagée par toutes les femmes, masquant ainsi les différences de statut économique et politique, voire les antagonismes qui les séparent. Le féminisme postmoderne approfondira cette critique à la fin des années quatre-vingt. Occultant souvent les rapports sociaux fondateurs des catégories de sexe, il reprochera tant aux féministes matérialistes que différencialistes, de fonder obligatoirement la catégorie femmes sur le partage de caractéristiques biologiques ou culturelles communes.

\section{L'articulation des rapports sociaux : Classe, genre, ethnicité, nation et "race".}

Ces travaux examinent, entre autres, l'articulation du sexisme et du racisme, dans les sociétés esclavagistes et coloniales mais aussi dans les situations très contemporaines et inégalitaires de l'immigration et du système-monde. Ils y analysent les inégalités qui séparent les femmes sur le plan économique, politique, culturel et idéologique. De nombreuses recherches, souvent de type qualitatif, comparent le travail rémunéré et non rémunéré des femmes de couleur et immigrantes à celui des blanches et des natives et évaluent leurs trajectoires socio-économiques respectives.

Alors que certains auteurs se penchent sur le processus de catégorisation luimême, d'autres tels Arthur Brittan et Mary Maynard (Sexism, Racism, and Oppression,
Oxford, Basil Blackwell, 1984, 236p.), rappellent que le sexisme, le racisme et le classisme sont des formes d'oppression qui, tout en possédant des histoires différentes, n'en agissent pas moins de façon interdépendante. Aussi faut-il s'abstenir de tracer des parallèles entre ces oppressions et d'en faire une simple somme arithmétique pour adopter un paradigme multidimensionnel qui met l'accent sur leurs interrelations. La dynamique des rapports de sexe dans un contexte institutionnel, la famille par exemple, varierait selon qu'on étudie des couples blancs ou noirs, de la bourgeoisie ou du prolétariat.

Pour ce qui est des polémiques entre ceux qui insistent sur l'existence d'un seul système de relations sociales incluant le genre, l'ethnicité et la classe (Floya Anthias and Nira Yuval-Davis, in association with Harriet Cain (1992), Racialized Boundaries. Race, Nation, Gender, Colour and Class and the Anti-racist Struggle, Londres et New York, Routledge, 226p) et ceux qui préconisent l'existence de systèmes analytiquement distincts et interdépendants (Stuart Hall, " Gramsci's relevance for the study of race and ethnicity ", Journal of Communication Inquiry, vol. 10, no 2 : 5-27,1986), elles n'ont pas encore trouvé réponse.

Ce débat n'est pas sans rappeler celui qui opposait, en France notamment, les féministes marxistes aux féministes matérialistes. Tandis que les premières mettent principalement l'accent sur la relation des femmes aux rapports de la production capitaliste, les secondes cherchent à théoriser le fondement matériel spécifique de l'oppression et de l'exploitation des femmes. Les assises et les conséquences de ce différend théorique sont concrètes et opèrent au plan politique. Quand on reconnaît l'existence de systèmes distincts de rapports sociaux, on est porté à chercher le fondement de chaque oppression, de classe, de sexe, d'ethnicité. Une telle démarche permet d'entrevoir les fondements matériels qui sous-tendent les catégories sociales, de dégager leurs intérêts communs et 
d'envisager, en dépit des différences bien réelles, les bases d'une action collective. Les coalitions de femmes en faveur de l'avortement et contre la pauvreté, de même que la collaboration de groupes féministes pour soutenir des femmes violées en temps de guerre de part et d'autre de la frontière ethnique, reconnaissent implicitement ou explicitement, l'existence d'un système distinct de relations sociales qui fonde leurs intérêts communs.

Quoi qu'il en soit, l'orientation théorique qui prévaut met l'accent sur la construction de catégories sociales distinctes et sur les liens qui s'établissent entre elles. Ce qui nous amène à examiner la manière dont s'imbriquent divers processus de différenciation et de hiérarchisation sociales.

\section{Hiérarchisation et différenciation sociales}

La place des femmes dans les rapports de sexe engendre leur relation spécifique à la production et à la reproduction de l'ethnicité. En tant que premières responsables de la socialisation des nouveaunés, elles contribuent, à l'intérieur d'une relation d'entretien corporel, affectif et des êtres humains, à I'humanisation et à l'ethnicisation de ces derniers (JuteauLee, Danielle " La production de I'ethnicité ou la part réelle de l'idéel ", Sociologie et sociétés, vol. 15, no 2, 1983: 39-54).

Le rapport des femmes à l'ethnicité passe d'abord et avant tout par leur contribution, spécifique et exigée, à la reproduction biologique et culturelle du groupe ethnique ou national. Cette contribution, de même que les sévices dont elles font l'objet en temps de paix comme en temps de guerre, s'inscrit dans les rapports de sexage, la classe des hommes exerçant un contrôle sur la classe des femmes, sur leur travail et sur leur corps. Les modalités du rapport des femmes à l'ethnicité diffèrent toutefois selon qu'elles appartiennent à un groupe eth- nique dominant ou dominé.

L'imbrication de ces rapports se manifeste diversement. La famille et la maternité sont placées au centre du discours nationaliste, qui défend la pureté de la “ race " et I'homogénéité du groupe. La représentation des femmes, qui est au cœur de l'imagerie nationale (la Marianne), sert à marquer les frontières et à définir l'identité du groupe. La “ question des femmes " se voit utilisée pour comparer et évaluer divers groupes ethniques. Bien que les femmes participent aux luttes de libération nationales, elles demeurent souvent privées des droits de la citoyenneté. Enfin, la conception de la féminité est indissociable de la construction de l'identité ethnique ou nationale (Éléni Varikas, " Sentiment national, genre et ethnicité ", Tumultes, no 11, Appartenances et ethnicités : 87-99, 1998), comme le montre la définition de l'anglicité à la période coloniale.

La reconnaissance des rapports de sexe fonde la critique de la vision androcentrique et essentialiste qui a longtemps caractérisé le champdes relations ethniques. Ce faisant, elle ouvre la voie à de nouvelles réflexions sur la constitution des collectivités ethniques et nationales et sur les rapports d'alliance entre les femmes dans l'établissement d'une politique dite transversale et transnationale (Caren Kaplan, Norma Alarcon, Minoo Moallem (sous la direction de), Between Woman and Nation. Nationalisms, Transnational Feminisms, and the State, Durham et Londres, Duke University Press, 1999, 407p). 


\section{Bibliographie}

Brubaker, Rogers (1996). Nationalism reframed. Nationhood and the national question in the new Europe, Cambridge, Cambridge University Press, 202p.

Guillaumin, Colette (1992). Sexe, Race et Pratique du pouvoir, Paris, Côtéfemmes, 239p.

Juteau-Lee, Danielle (1983). “ La production de l'ethnicité ou la part réelle de l'idéel ", Sociologie et sociétés, vol. 15, no 2: 39-54.

Sollors, Werner (1986). Beyond Ethnicity. Consent and Descent in American Culture, New York, Oxford University Press, 294p.

Varikas, Éleni (1998). Sentiment national, genre et ethnicité. Tumultes, no 11, Appartenances et ethnicités : 87-99.

Yuval-Davis,Nira (1997). Gender and Nation, Londres, Sage, 157p. 